\title{
Lifetime Enhancement in Wireless Sensor Network using Modified Energy Sheduling Algorithm
}

\author{
Srinath $\mathrm{V}^{\# 1}$, Arunmozhi S.A ${ }^{* 2}$ \\ ${ }^{\#}$ PG Scholar \& Associate Professor \& Department of ECE \\ Saranathan College of Engineering, Trichy.
}

\begin{abstract}
Energy consumption and network lifetime are the two important factors for wireless sensor networks. In energy efficient the sensor node finds a suitable path for transferring its information. Power consumption is also one of the factors which reduce the lifetime. Aim of sleep scheduling algorithm is to analyse the behaviour of nodes in the network. To save the energy consumption the modified energy scheduling with wake up protocol have been proposed. The scheduling scheme which decides the eligibility criteria for each node, whether the node is an active or a sleep state. The protocol which adjust the sleep time of node in order to match the network even in harsh environment. The sleep scheduling algorithm increases the throughput and delivery ratio To verify and evaluate, various simulation parameters were considered to extend the network lifetime using $n s-2$ simulator.
\end{abstract}

Keywords - Sensor network, node scheduling, coverage area, blind point

\section{INTRODUCTION}

A wireless sensor network is a set of sensor nodes organized into different types of networks like tree, mesh etc. A Wireless Sensor Network (WSN) contains different hardware part for sensing and computation .WSN devices are restricted in their energy, processing, and communication capabilities. The sensor nodes are work by the battery which is placed in remote area so it is not very easy to replace and recharge the battery after deployment. Thus, the look and development of low-energy algorithms and protocols are essential for sensor networks.

Especially, the energy consumption of data transfer between nodes strongly dominates other node functions such as sensing and processing. A wireless sensor network is a combination of many sensing nodes called wireless sensor nodes where each node is small in size, less in weight and portable in nature. Each sensor node contains a transducer, microcomputer, transceiver and power source for communication. Generally the nodes are equipped with power-constrained batteries, which are often difficult, expensive and impossible to replace once the nodes are deployed. The challenge of sensor network protocol is energy conservation \& limited bandwidth. The energy consumption by a node depends on its state. The four states available for each node is transmit, receive, idle (state where no messages are transmitted) and sleep (state where no communication takes place).

Lifetime of the network is one of the most critical issues in Wireless sensor networks (WSNs) since most sensors are equipped with nonrechargeable batteries with restricted energy. WSN are widely used to detect movement, temperature changes, precipitation etc.

In high density sensor network, only a subset of nodes is to sense and communicate, where the remaining node is in sleep mode. From time to time subset of node is changed in order to reduce energy consumption, and also it helps to reduce the packet collision and delay .The energy level of all nodes must update the information received from source node to their neighbours and hence it achieves the significant reduction in number of messages exchanged.

Thus the coverage and energy consumption are two metrics in determining the network quality. The quality of service deals with device calibration, environment monitoring and exposure. Exposure can be defined as the measurement of coverage of a region in terms of detection ability for a moving target. WSNs are facing a number of challenges due to scarce energy resources, dynamic nature of topology and a number of other network elements like number of sensor nodes deployed, method of deployment, communication and sensing ranges associated with sensor nodes.

\section{LITERATURE SURVEY}

Wireless sensor network consists of several resource- rich nodes used for data relaying and a large number of energy constrained[3]wireless sensor nodes. Generally sensor nodes are deployed to monitor targets. The targets are redundantly covered by many sensors that has to find a maximum number of set covers $\&$ each active sensor is connected to at least one source node. A sensor can participate in multiple set covers, but the total energy spent in all sets are constrained by the battery capacity. The probing environment and adoptive sleeping was 
considered[4] using a very large number of small sensor with short battery life time. It extends the network life time by maintaining a necessary set of working nodes and turning off redundant ones.

This operations are based on individual nodes observation of the local environment and do not require any node to maintain other neighbour node state. It poses a high degree of robustness in the presence of both node power depletion and unexpected failures. So the stimulation show that it can maintain an adequate working node density to a node failure and it can maintain constant over head level under various deployment conditions ranging from sparse to dense node [11] provides security against various attacks and help to secure the network for a prolonged lifetime.

The coverage problem [8] divided into three types namely target coverage, area coverage and barrier coverage. The main goal of area coverage is to provide a full coverage over an area by consuming less energy state. Since the sleeping state is the least energy consumption, it is preferred to solve this coverage problem. To divide the [2]nodes into disjoint sets such that every state can individually monitoring the task. These set are successfully activated and all the nodes not belonging to the active set will be in the sleep state.

Each location in the physical space within the sensing range must have one sensor. Each sensor node alternates between active and sleep states to conserve energy with an average sleep period longer than the active period. The dynamic[5] change in topology has potentially affect the operation and performance of the network. This is compensated by adding redundancy in the sensor deployment.

In this paper the relationship between the reduction in sensor[6]duty cycle and the required level of redundancy to be measured. In particular, two types of mechanisms are used namely the random sleep schedule where each sensor keeps an activesleep schedule which is independent of another, and the coordinated sleep type where sensors coordinate with each other. Both types are studied within the context of providing network coverage. Redundant sensors affect [9] the communication between nodes and minimum number of deployed sensor will guarantee the coverage of the region. Coverage must able to address the selection of a sensor nodes to cover the border of a coverage region.

Adequate coverage[7] is one of the main problems for sensor networks. The effectiveness of distributed wireless sensor networks highly depends on the sensor deployment scheme. Given a finite number of sensors, optimizing the sensor deployment will provide sufficient sensor coverage and save power of sensors for movement to target location to adequate coverage. In many working environments, for getting good coverage, we must able to move sensors to adequate places.

\section{EXISTING METHOD}

Coverage node scheduling is localized and separated into rounds. Coverage preserving nodes scheduling algorithm is localized and divided into rounds. Each round starts with self-scheduling followed by sensing. In this the off-duty eligibility rule is used by the nodes for investigation. Based on this rule each node verifies its sensing area covered by their sensing neighbours. If it satisfies the above rule the eligible nodes turned off their communication and sensing process, whereas the other nodes called active nodes will perform the monitoring task. To avoid the occurrence of blind point the priority is assigned to nodes.

A localized probing-based self-scheduling algorithm is an approach where a node goes back to the sleep state if it receives at least one reply to its probation message, otherwise it remains active. The problem is that algorithm does not guarantee the coverage of the target area. To handle the coverage problem, coordinated sleep scheduling algorithm is proposed. In this algorithm, a node assumes multiples roles namely a sponsor and a regular node. Periodically, each node determines the set of its sponsors covering its sensing area and send a request message to each sponsor. The eligible node receiving confirmation from the sponsor node and enter the sleep state. The self scheduling phase in the network which detect the source node and ready to transfer the data from source to destination .

\section{PROPOSED METHOD}

Modified Energy scheduling algorithm aims to provide a full coverage over an area by minimizing the number of active nodes. To meet these objectives a sub set of active nodes is periodically selected. Based on energy scheduling each node decides by its own whether it must be taken its energy level, one hop neighbourhood information the coverage rate of its sensing area. The main aim is to achieve a balanced energy consumption among sensor nodes and hence exchanging a minimum amount of messages for node scheduling by using minimum active nodes. Hence the massive use of sub set of nodes may induce their premature death. In our proposed work the energy scheduling algorithm works on the basis of energy conservation and network stability

Each node contribute to the target area only through its sensing region. Thus each node guarantee the full coverage of its sensing area by a sub set of working neighbor before entering its sleep state, then the coverage area will be preserved even after the deactivation of redundant nodes .Thus the blind point occur in the coverage region. 


\section{OCCURENCE OF BLIND POINT}

Wireless sensor network which effectively cover the target monitoring region. The radius of the sensor node is fixed, but the random deployment of nodes may produce blind spot may degrade the performance of the network. To avoid the blind point, priority must be introduced between nodes. The priority avoids the use of additional messages and allows to remain robust. As energy consumption is one of the major constraints and establish balanced energy among the nodes. Thus the most exhausted nodes should have the lowest order compared to its neighbour. Consequently, they should have the highest priority to enter sleep state in order to preserve their energy.

\section{STEPS FOLLOWED IN ENERGY SCHEDULING ALGORITHM:}

\section{HELLO STEP \\ 2. ELIGIBLITY STEP}

\section{Hello Step:}

At the beginning, each node transmits to its neighbouring nodes an hello message along with its ID and its remaining energy. As only the nodes within a sensing range are consider for verification of SA coverage. This minimize the energy consumption. When receiving a hello message, the receiver node check the availability of region and transmits the information. Based on the information the eligibility for each node is verified.

\section{Eligibility step:}

After the time out, of hello message the eligibility step takes place. The proposed algorithm introduces priorities between the nodes. Nodes with minimal residual energies will have a greater priority to turn off. If the sensing area is covered by the union of the sensing area of the nodes belonging to its sleep state. on the other hand if the sensor area is not covered, the node can directly decide to be active state such decision does not minimize the number of active nodes. Hence the node sends an acknowledgement request to the selected node for verifies its sensing area and availability of region and it waits for the response from the source node. Then the source node finds the correct path for its neighbouring node to transmit the information .

\section{FLOWCHART OF MODIFIED ENERGY SCHEDULING}

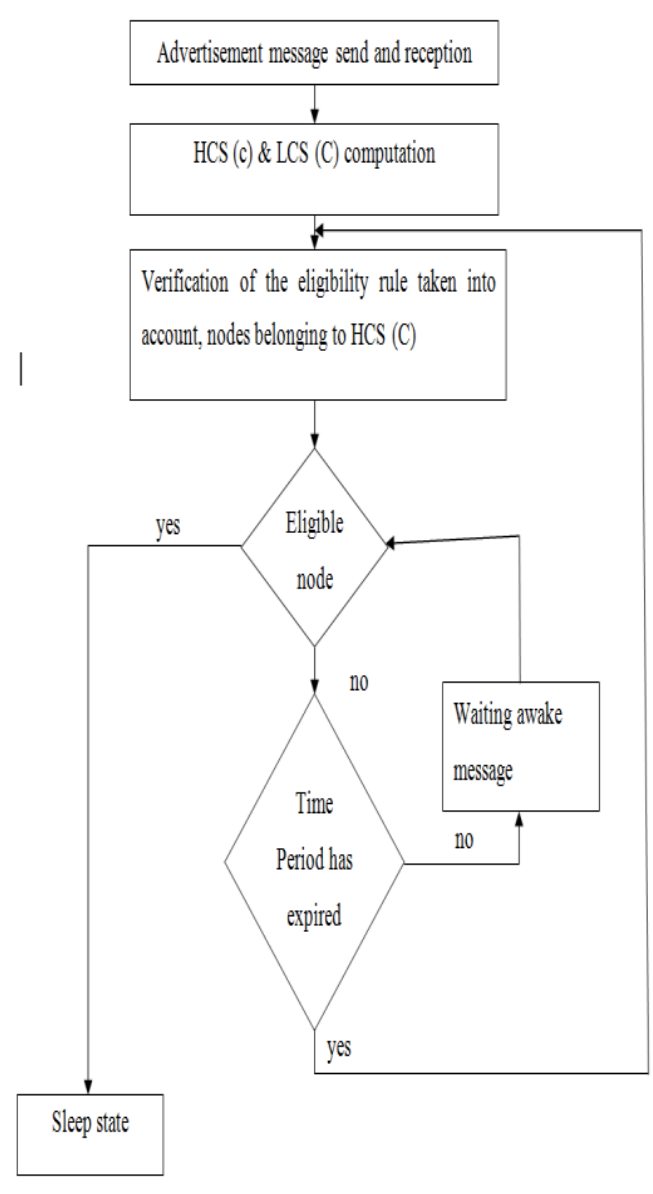

The node send an hello message to its neighboring node which it contains the current ID and residual energy. After sending the message ,each node verifies its sensing region and broadcast the hello message to its neighbouring nodes which is nearer to its coverage area.

The verification of eligibility rule taken into account and hence if the node is eligible then it directly assign it until the particular time period has expired. This process is repeated until it checks each and every node in the network. This algorithm is particularly used for saving energy consumption in the network.

After the verification of eligibility rule, the nodes in the network are verified and send an acknowledgement to a selected source node. Each selected source node sends an route request packet to its neighbouring node and finds the suitable path for sending the packet .

Here the offline nodes are often occurred is to kept in a sleep state in order to conserve the energy and hence if the node satisfies its eligibility then it directly promote to the sleep state. on the other hand if the node is not eligible it waits for the hello message and hence the node is in active state. 
Thus the procedure is repeated for all nodes in the network until the each node satisfies the eligibility criteria. Hence the node with minimal residual energy take place in the list for saving the lifetime,

\section{COVERAGE SENSING AREA}

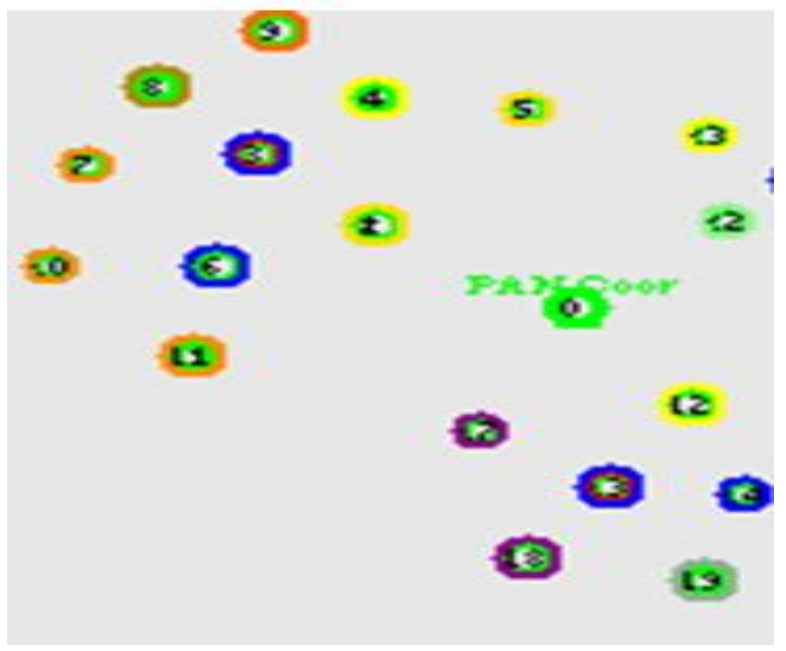

SCENARIO 1:

In these the nodes considers the eligibility verification of sensing area and hence the node 3 and 6 in the sensing area has greater priority than the shaded one. The remaining nodes have a lower priority than the node 3 and 6 and hence each node in the coverage sensing verifies with its neighbouring node and then checks for the eligibility criteria. Thus the residual energies of each node is analyzed and then it continues its processing stage. Thus the node is in the union of sensing area then the source node is directly assigned into the sleep state.

\section{SCENARIO 2:}

In these, the eligibility verification of nodes which broadcast the message to its neighbouring node and hence each time the source node verifies the union of sensing area and hence the decision is based on the priority and the node which is not in the union of coverage region and it is not eligible directly to assign into the sleep state. Hence the subset of each node in the network is in the active state and hence the node in with its availability of region allows the minimal energy in the network. Thus the balanced energy consumption is maintained in the network.

\section{RESULT AND DISCUSSSION}

The results are shown in the graphs. The calculation of the performance is calculated on the basis of these parameters. These are through put and packet delivery ratio. These parameter is measured using network simulator.

First the random deployment of nodes were shown in the network animator. There are 40 nodes which get randomly deployed so that each node is to transfer the data to its neighbouring nodes and also verifies the union of sensing range. Thus the $\mathrm{X}$ graph determines the analysis of nodes behavior on the initial stage of the process.

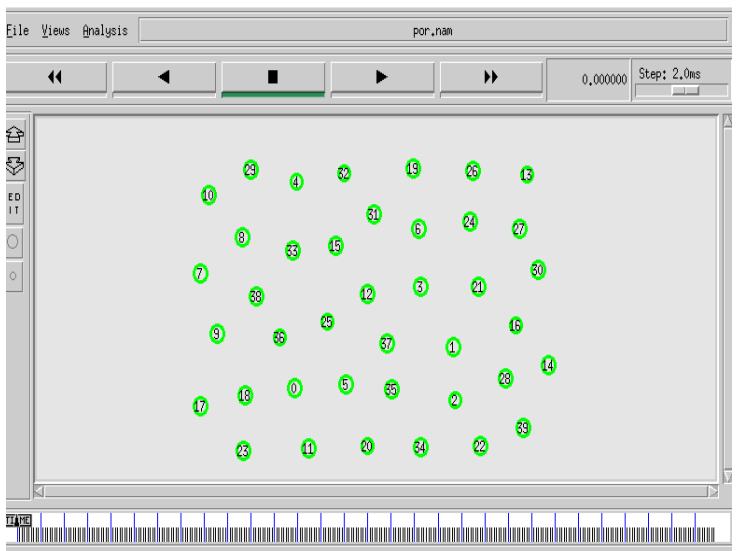

Fig 1: Random Deployment of nodes

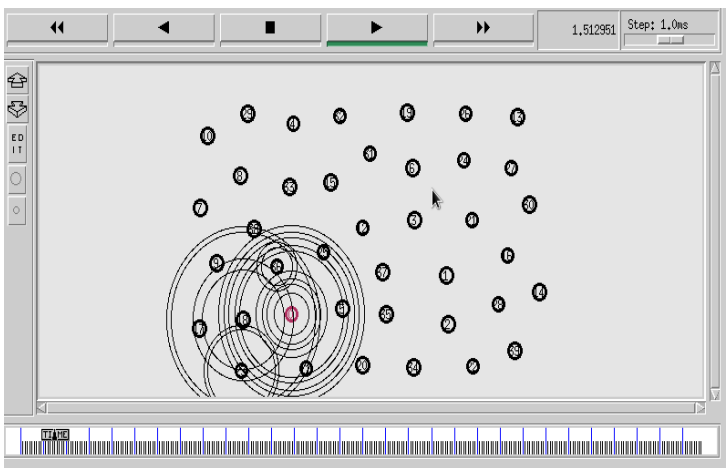

Fig 2: Broadcast message to the neighbour

After the deployment, each nodes broadcast the hello message to its neighbour nodes and hence the selected node which fall on its union of sensing region is easily identified and transmit the data until the next hop occurs. Thus the node also check the coverage of the target region and minimize the number of redundant nodes in the network.

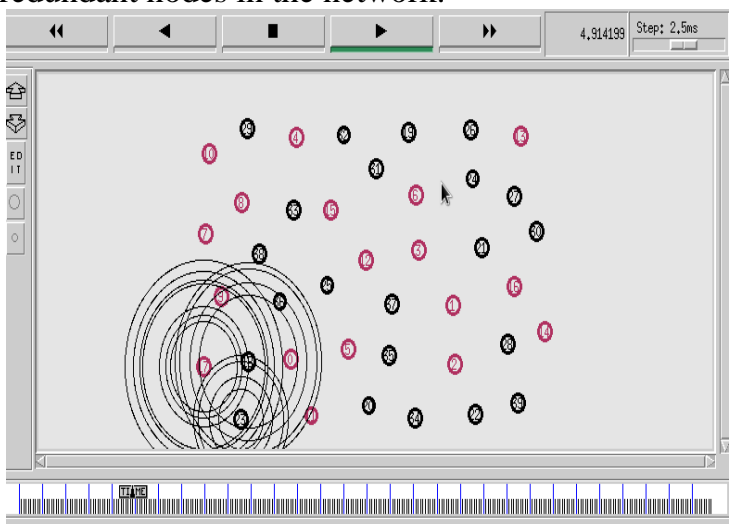

Fig 3: Node send a hello message

Node broadcast the message to its neighbour After Broadcasting the message, each node in the network sends an hello message to its neighbour and verify the sensing area of the source node which 
covers the coverage region and hence it also send an acknowledgement to a selected node and it waits for a response route request packet .

Each Selected node sends an route request packet to its neighbouring node and find the correct path for sending the packet from source to destination. so the packets from each node are kept in a sleep mode to conserve the energy.

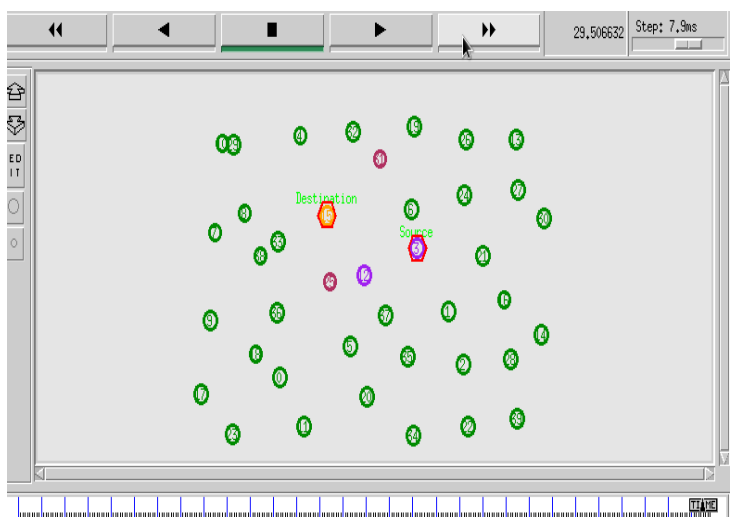

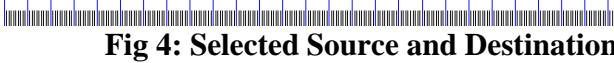

\section{PERFORMANCE METRICS}

In this three important performance metrics are used. These parameters are compared with existing one and hence modified scheduling algorithm provides better throughput and packet delivery ratio.

\section{A. Throughput}

Throughput is the rate of successful message delivered over a communication channel. It is obtained by using formula.

Throughput=(total no of bytes received/simulation time $)$

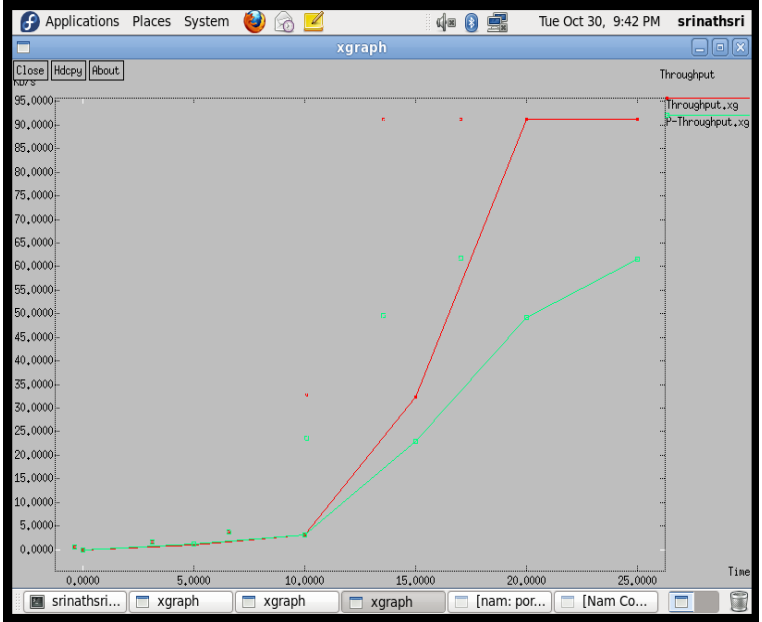

Fig 5: Throughput $x$-graph

\begin{tabular}{|l|l|l|}
\hline Time (Kbps) & $\begin{array}{l}\text { Localized Self } \\
\text { scheduling } \\
\text { algorithm }\end{array}$ & $\begin{array}{l}\text { Modified energy } \\
\text { scheduling } \\
\text { algorithm }\end{array}$ \\
\hline 0 & 0 & 0 \\
\hline 5 & 1.12 & 1.68 \\
\hline 10 & 12.45 & 31.64 \\
\hline 15 & 45.62 & 64.23 \\
\hline 20 & 72.25 & 82.0 \\
\hline
\end{tabular}

Table 1: Throughput Comparison

From the simulation result, the comparison of throughput value is calculated, and hence the throughput value of modified energy scheduling algorithm is better than the existing method in the case of heavy number of nodes.

\section{B. Packet delivery Ratio}

Packet delivery fraction, the performance increases with increase in the value, it means higher the value better its performance and it is calculated by using formula given by

packet delivery ratio $=($ no of received packets/no of delivered packets)*100

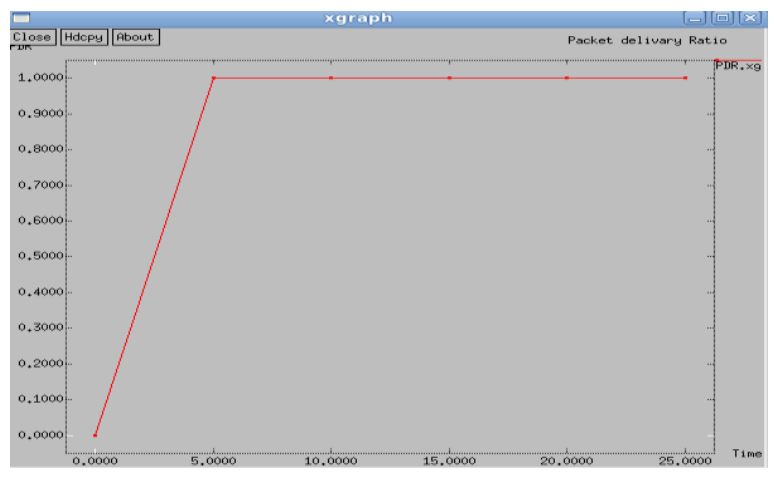

Fig 6: Packet delivery ratio

Table 2: Packet Deliveery ratio comparison

\begin{tabular}{|c|c|c|}
\hline Parameters's & $\begin{array}{l}\text { Hybilil Scheeluling } \\
\text { Algoithun }\end{array}$ & ERGS \\
\hline Source Type & TCPIDP & TCP/UDP \\
\hline Routing Protocol & DSDV & DSDV \\
\hline Rate & 10kbops & 10kbpss \\
\hline Packet delivery ratio & 40kbops & 47 kboss \\
\hline
\end{tabular}


From the comparison the packet delivered ratio of energy scheduling algorithm is much better than the existing method.

\section{CONCLUSION}

In this paper, Energy efficiency of nodes is maintained by Dynamic selection of Nodes using scheduling algorithm. Modified Energy scheduling with wakeup protocol have been proposed for energy minimization by scheduling sensor nodes activity to switch on only a subset of sensors that covers area while all other sensors are turned off. Our mechanism maximizes the lifetime while ensuring the full coverage of the target area of the obtained configuration. The main feature of the Modified Energy scheduling algorithm is to balance the remaining energy of the nodes. This has contributed to preserve the coverage over a monitored area and consumes less energy. The second main feature consists in avoiding negotiation phases, as decision to enter sleep state uses a computed priority based on a one-hop neighbourhood. This contributes not only to preserve the coverage but also occurrence of blind point is avoided in order to enhance the lifetime of a network.

\section{REFERENCES}

[1] Ammari H.M. and Giudici J. (2009), ' On the connected kcoverage problem in heterogeneous sensor nets: the curse of randomness and heterogeneity'. ICDCS 09: Proc. 2009 29th IEEE Int. Conf. on Distributed Computing Systems, pp.265272.

[2] Arboleda L.M.C. and Nasser N. (2006), 'Comparison of Clustering Algorithms and Protocol for Wireless Sensor Networks,' Proc.Canadian Conf. Electrical and Computer Eng, pp.1787-1792

[3] Arms Chris Townsend S. (2004), 'Wireless Sensor Network: principles and applications,' in Wilson J.S. (Ed): 'Sensor Technology Handbook,' pp.439-450.

[4] Heizelman W.R. and Chandrakasan A. (2008), ' Energy Efficient Communication Protocol for Wireless Micro Sensor Networks,' Proc. IEEE Hawaii Int. Conf. System Science, Jan 2008.

[5] Hwang S.F. and Dow C.R. (2006), 'A Cluster-Based Coverage-Preserved Node Scheduling Scheme in Wireless Sensor Networks,' Proc. Third Ann. Int. Conf. Mobile and Ubiquitous Systems: Networking and Services, pp.1-7.

[6] Karl H. and Willing A. (2005), 'Protocols and Architectures for Wireless Sensor Networks. 'International conference on communication networking and system ,pp.234-245.

[7] Reason J.M. and Rabies J.M. (2004),' A study of Energy Consumption and Reliability in a Multi-hop Sensor Network'.

[8] Thai M.T et al. (2008),' Coverage Problems in Wireless Sensor Networks: Designs and Analysis,' Int. J. Sens. Network, pp.191-200.

[9] Wang X et al. (2005),' Integrated Coverage and Connectivity Configuration in Wireless Sensor Networks,' pp.28-39.

[10] Arunmozhi, SA \& Venkataramani, Y (2010), 'A Flow Monitoring Scheme to Defend Reduction-of-Quality Attacks in Mobile Ad-hoc Networks', Information Security Journal: A Global Perspective, vol.19, no. 5, pp. 263-272.
[11] Arunmozhi, SA \& Venkataramani, Y 2011, 'A new defense scheme against DDoS attack in MANETs', Communications in Computer and Information Science, vol.133, pp.210-216. 